\title{
FLOK SOCIETY EN ECUADOR 1.0. LA TRANSICIÓN HACIA LA ECONOMÍA SOCIAL DEL CONOCIMIENTO A PIE DE OBRA
}

\author{
David Vila Viñas \\ Investigador principal proyecto Buen Conocer / FLOK Society. Becario postdoctoral Prometeus (SENESCYT, \\ Gobierno de Ecuador).
}

\begin{abstract}
Resumen
Describe los procesos, los colaboradores involucrados y la repercusión del desarrollo del proyecto ecuatoriano "Sociedad FLOK - Free Libre Open Knowledge Society", con miras a la construcción de planos para fortalecer la economía social del conocimiento social común y abierto. El proyecto dio lugar a la Cumbre del Conocer Bien en mayo de 2014 en Quito, con diferentes actores e instituciones sociales. Sus resultados serán publicados en un libro con las principales directrices disponibles en un número especial del "Journal of Peer Production". Durante la elaboración del proyecto y en la Cumbre se asistió a una intensa participación de varias instituciones, entre ellas de la comunidad internacional. La inicialmente difícil participación de la sociedad civil que por las barreras del idioma fue superada a partir de la idea de "Bueno saber cómo habitar Bien Vivir", marco político ecuatoriano desde 2008, y por los esfuerzos de instituciones, tornando posible aumentar la participación de la sociedad civil. De este modo, a través de una red participativa, las discusiones que produjeran contenidos en 190 documentos iniciales fueron publicadas, lo que llamamos "propio contenido". Su impacto y discusión aparecieron en los medios de comunicación tradicionales, un total de 130, lo que llamamos "contenido en medios", en las discusiones en los "medios de comunicación de redes sociales " y de diversas acciones de comunicación y equipo FLOK . Por último, también alcanzamos lo que llamamos redes de territorios que hibridaran territorios digitales y geográficos en las reuniones \# Beer2peer en los bares de Quito. A pesar de alta visibilidad dada al contenido propuesto, la eficacia de las recomendaciones de política y de proyectos productivos aún tiene bajo rendimiento por el pequeño número actual de participantes en el equipo. Todavía hay cuellos de botella como la necesidad de programas de capacitación, programas piloto y tácticas para promover los ya posibles planes y profundizar los iniciados.
\end{abstract}

Palabras-clave

FLOK. Economía del conocimiento en Ecuador. Movilización de redes. Proyecto P2P en Ecuador.

\section{A SOCIEDADE FLOK NO EQUADOR 1.0: A TRANSIÇ̃̃O LOCAL PARA A ECONOMIA SOCIAL DO CONHECIMENTO EM MARCHA}

\begin{abstract}
Resumo
Descreve processos, colaboradores envolvidos e repercussão da elaboração do projeto equatoriano "FLOK Society _ Free Libre Open Knowledge Society", com vistas à construção de planos que visem reforçar a economia social do conhecimento social comum e aberto. Projeto ensejou a Cúpula do Bem Conhecer, em maio de 2014 em Quito, contando com diversos atores e instituições sociais, e seus resultados serão publicados em um livro, estando as principais diretrizes disponíveis em um número especial do "Journal of Peer Production". Durante a elaboração do projeto e na Cúpula contou-se com uma intensa participação de diversas instituições, inclusive da comunidade internacional. Inicialmente a difícil participação da sociedade civil, pelas barreiras de língua, foi contornada a partir da ideia de "Bem Conhecer como habitat do Bem Viver", marco político equatoriano desde 2008 e pelos esforços de instituições, sendo possível reforçar a participação da sociedade civil. Assim, através de uma rede participativa, as discussões que produziram conteúdos em 190 documentos iniciais foram publicados, que chamamos "conteúdo próprio". Sua repercussão e discussão apareceu nas mídias tradicionais, num total de 130, que chamamos de "conteúdos de mídias", nas discussões em "mídias de redes sociais" e a partir de diversas ações de comunicação da equipe do FLOK. Finalmente, também chegou ao que chamamos redes territórios que hibridizaram os territórios digitais e geográficos nos encontros \#Beer2peer nos bares de Quito. Apesar da ampla visibilidade dada aos conteúdos propostos, a eficácia das recomendações políticas e dos projetos produtivos ainda apresenta baixa concretização pelo atual reduzido número de participantes na equipe. Existem ainda gargalos como a necessidade de planos de formação, programas pilotos e táticas para levar adiante os planos já possíveis e aprofundar os processos iniciados.
\end{abstract}

Palavras-chave

FLOK. Economia do conhecimento no Equador. Mobilização de redes. Projeto P2P no Equador. 


\title{
FLOK SOCIETY IN ECUADOR 1.0. THE TRANSITION TO THE SOCIAL ECONOMY OF THE LOCAL KNOWLEDGE
}

\begin{abstract}
Describes processes, collaborators involved and impact of the development of the Ecuadorian project "Flok Society_FreeLibre Open Knowledge Society" with a view to building plans to strengthen the social economy of the common and open social knowledge. Project gave rise to the Summit Conocer Bien in May 2014 in Quito, with different actors and social institutions, and their results will be published in a book, with the main guidelines available in a special issue of the "Journal of Peer Production". During the project design and the Summit was attended up with an intense participation of several institutions, including the international community. The initially difficult participation of civil society, by language barriers, was dealt from the idea of "Well know as habitat of the Good Life", Ecuadorian political landmark since 2008 and the institutions of efforts, it is possible to increase the participation of civil society. Thus, through a participatory network, the discussions that produced content in 190 initial documents were published, which we call "own content". Its impact and discussion appeared in traditional media, a total of 130, which we call "media content" in discussions "social networking media" and from diverse and communication actions offlok team. Finally, also reached what we call territories networks hybridized digital and geographical territories in meetings \#Beer2peer in the bars of Quito. For the sake of high visibility given to the proposed content the effectiveness of the policy recommendations and productive projects still has low achievement by the current small number of participants in the team. There are still bottlenecks as the need for training programs, pilot programs and tactics to further the already possible plans and deepen the initiated. Keywords

Flok. Knowledge economy in Ecuador. Mobilization networks. P2P project in Ecuador
\end{abstract}

Buen Conocer - FLOK Society (Free Libre Open Knowledge Society) es un proyecto desarrollado en Ecuador, aunque con notables repercusiones internacionales, destinado a elaborar de manera colaborativa, entre organizaciones movilizadas y especializadas de la sociedad civil, expertos académicos y representantes de instituciones públicas, normas, políticas públicas y proyectos productivos dirigidos a reforzar la economía social del conocimiento común y abierto (ESC). Ecuador ha sido el contexto en que desarrollar este proyecto porque su economía pretende transitar desde una base exportadora de productos primarios de escaso valor añadido y de alto impacto ecológico (ello incluye tanto al petróleo como a la palma o los camarones) e importadora del resto, hacia una economía capaz de participar en esos ciclos de mayor valor añadido, marcados por la relevancia del conocimiento, y de reducir así su dependencia respecto a la explotación de esos recursos insustituibles. Para un país con las condiciones de Ecuador, la transición hacia el mayor peso de una economía basada en el conocimiento, dentro de una noción amplia que abarcaría desde el desarrollo de software hasta la proliferación de emprendimientos turísticos, está en marcha de manera difícil de revertir. Sin embargo, la cuestión para un proyecto como FLOK Society no es impulsar la implantación de una economía del conocimiento sin más, de tendencia por lo demás hegemónica en todo el globo, sino favorecer que esa transición se haga en condiciones de máximo acceso, utilidad social y sostenibilidad. 
Con este objetivo, el proyecto ha tenido dos etapas en esta primera fase. Una, basada en el documento de diseño del proceso, desde noviembre de 2013 a 30 de junio de 2014 (fecha en que terminó el trabajo de los equipos de investigación, comunicación y administración contratados), elaborado por Daniel Vázquez y Xabier Barandiaran, que concentró la mayor parte de esfuerzo económico y humano del proyecto, incluidas un sinfín de relaciones con movimientos sociales y entidades públicas, desembocando en la Cumbre del Buen Conocer, celebrada en Quito entre el 27 y 30 de mayo de 2014. Y una segunda, que se extenderá hasta marzo de 2015, centrada en el proceso de elaboración, edición y visibilización de las propuestas finales en distintas áreas bajo la coordinación de David Vila. Para hacer una valoración general de esta fase conviene distinguir, aunque sean cuestiones muy interconectadas, entre los aspectos sustantivos (propuestas para una ESC) y los metodológicos (procesos de participación, efectuación de las propuestas, etc.).

Respecto a las propuestas, el contenido general del plan de investigación, así como algunos lineamientos para la transición hacia la economía social del conocimiento pueden verse en el documento de Michel Bauwens, director de investigación hasta julio de 2014, que aludía a las siguientes líneas y materias, cuyos documentos de política pública, en sus distintas versiones pueden consultarse aquí:

http://floksociety.org/pad/p/indice_Documentos_Investigaci\%C3\%B3n_Buen_Conocer

1. Desarrollar el talento humano.

1. Recursos de educación abierta

2. Ciencia abierta

3. Cultura libre

2. Capacidades productivas orientadas hacia los comunes.

1. Agricultura abierta y sostenible

2. Biodiversidad

3. Energía distribuida

4. Fabricación distribuida y diseño abierto

3. Institucionalidad de la economía social del conocimiento.

1. Conocimiento social y economía social.

2. Política pública para la economía social.

3. TIC, datos abiertos y gobierno abierto.

4. Política pública para el Partner State.

4. Infraestructuras técnicas abiertas. 
1. Software libre.

2. Hardware libre.

3. Conectividad.

4. Ciberseguridad (integrado finalmente en los anteriores).

5. Infraestructuras comunes del conocimiento en las comunidades.

1. Territorialización del trabajo cognitivo.

2. Saberes ancestrales y tradicionales en la economía social del conocimiento.

Este proceso, que incluye a una gran cantidad de autores/as y colaboradres/as en sus distintas versiones, culminará con la edición de un libro con los principales resultados y de un número especial en la Journal of Peer Production. Debido al objetivo general del proyecto, la investigación se dirigió hacia distintas áreas que se consideraban estratégicas para la orientar la transición hacia el conocimiento común y abierto. La Cumbre del Buen Conocer reprodujo esa estructura discutiendo, validando y reelaborando las propuestas específicas en 14 áreas, correspondientes a las 14 mesas de trabajo (http://floksociety.org/wpcontent/uploads/2014/05/Declaraciones-Buen-Conocer.pdf ). El resultado de la mayor parte de los documentos es realmente interesante y contiene análisis y propuestas eficaces, no solo para Ecuador, sino para la implementación de una ESC en distintos contextos de América Latina y el mundo. Uno de los principales aprendizajes del proceso es que, cuando se pretende hacer efectiva una transición hacia la ESC en un contexto determinado (un país admite la innovación pero nunca es un campo para experimentos), la exigencia en términos de especificidad, realismo, eficacia, concreción y escala de las propuestas es máxima. Casi todos los agentes con los que hemos dialogado en este tiempo repiten: "estamos de acuerdo en este objetivo de la economía social del conocimiento común y abierto pero ¿cómo lo hacemos aquí y ahora?".

El otro rasgo fundamental del proceso FLOK ha sido la participación. Por una parte, ésta incluía al proceso de investigación. Obviamente los documentos de política pública tienen detrás a distintos autores pero, en casi todos los casos, el contenido es fruto de distintos procesos de discusión con expertos y activistas en la materia y con responsables intermedios de las instituciones públicas competentes para llevar a cabo sus propuestas, así como de un proceso de valoración desarrollado en el wiki, en las listas de correo, en la plataforma de comentarios y en las mesas de trabajo de la Cumbre del Buen Conocer, que contó con más de 200 invitados comprometidos durante tres días de trabajo colaborativo.

Así y en términos generales, se valora positivamente la alta participación en distintas 
fases del proceso. La involucración inicial de la comunidad internacional fue alta, con un pico a partir del lanzamiento de la "Carta abierta a los trabajadores del procomún". Al momento de iniciarse la cumbre en mayo, los indicadores registrados eran los siguientes:

Presencialmente:

- Talleres Economía Social del Conocimiento: 25 talleres en 24 provincias, + 1000 participantes

- Reuniones de trabajo: 68 reuniones con 47 instituciones diferentes.

- En la cumbre: $+\mathbf{1 9 6}$ personas de $+\mathbf{1 0 0}$ instituciones, asociaciones, comunidades.

- Talleres diversos puestos en marcha con colectivos locales, como el titulado Guerrilla Comunicación en El Diferencial (con Bernardo Gutiérrez) o los 3 seminarios Amawta celebrados en el IAEN.

- A través de la red:

- Web principal: 85.450 páginas vistas, 190 noticias generadas.

- Wiki: 46 secciones de contenido, 4.092 ediciones, 226.668 visitas.

- Mumble: +46 personas han participado en reuniones de coordinación.

- Co-ment: 949 comentarios a los documentos con 140 participantes.

- Pads: 111 documentos colaborativos abiertos para coordinar actividades

- Listas de correo: 2.234 mensajes y 137 participantes

TOTAL ESTIMADO: $\mathbf{+ 1 . 5 0 0}$ personas han participado activamente en FLOK Society

A lo que hay que añadir la participación interinstitucional, con un total de 45 entidades participantes en el proceso, 27 de ellas ecuatorianas. Algunos ejemplos:

- Colectivos/Asociaciones ecuatorianas: ASLE, Usuarios Digitales, Coordinadora de Movimientos Sociales Por la Democracia y el Socialismo, Wikimedia Ecuador, Creative Commons Ecuador, Radialistas, Diabluma, Red de Innovación Educativa (RIE), Hackem, ECStartups, Sociedad de Divulgación Científica Quinto Pilar, Infodesarrollo, Colectivo Dogma, Fab Lab Cuenca, El Diferencial etc.

- Colectivos/Asociaciones internacionales: Ártica (Uruguay), P2PFoundation (Bélgica), SurSiendo (México), Universidad Nómada (España), Código Sur (UruguayArgentina), Interdisciplinar (Brasil), La Casa Tomada (El Salvador), Wikimedia México, Parque Explora (Colombia), Mapping the Commons (España/Brasil), Global Commons Alliance, etc. 
- Instituciones nacionales: IAEN, SENESCYT, MCCTH, INER, Cancillería, SNAP, SENPLADES, MINTEL, Alcaldía de Sigchos, Ministerio de educación, El Diferencial, Centro de Arte Contemporáneo de Quito, CIESPAL, IEPI, etc.

- Instituciones internacionales: Secretaría general de Presidencia de la República (Brasil), Participa.br (Brasil), Universidad Austral (Chile), Berkeley (EE.UU.), UPV/EHU (País Vasco, España), Universidad Federal de Minas Gerais (UFMG, Brasil), etc.

Sin duda, el ámbito de participación más difícil de movilizar ha sido el de la propia sociedad civil ecuatoriana, debido a la composición internacional, a la lengua y al propio imaginario inicial del proyecto, así como a la identificación con el mismo Estado ecuatoriano. Finalmente la animación de un imaginario más próximo, basado en la idea del Buen Conocer (Sumak Yachay en quichua), como hábitat del Buen Vivir, marco político adoptado por la sociedad ecuatoriana en el pacto constituyente de 2008, así como la horizontalización de la relación entre instituciones públicas y movimientos sociales americanos han permitido reforzar este plano de participación. Además, se han seguido las siguientes acciones concretas:

1. Elaboración de un documento de investigación sobre saberes ancestrales y tradicionales elaborado por intelectuales indígenas, donde cabe destacar la incorporación final de cinco intelectuales indígenas en la investigación y

2. Celebración de una mesa de discusión dedicada a esta temática y reelaboración de las recomendaciones sobre política pública a partir de los resultados de la mesa de discusión a través de un proceso colaborativo que ha implicado a más de una decena de personas expertas en el tema y bajo la edición de Juan Manuel Crespo.

3. Transversalización de este enfoque, que al menos se constata en los documentos sobre biodiversidad, agricultura, territorialización del trabajo cognitivo y cultura.

4. Visitas científicas del equipo de investigación, comunicación y coordinación a proyectos comunitarios urbanos y rurales, con el objetivo de alimentar los documentos y de trabajar conjuntamente en el diseño de algunos proyectos.

5. La organización Infodesarrollo realizó un conjunto de talleres en las 24 provincias ecuatorianas que involucró a más de 1.000 personas. A través de métodos de educación popular, se discutió la noción de economía social del conocimiento en los distintos territorios, con vistas a concretar la investigación. Algunos de los talleres desarrollados dieron lugar a grupos de trabajo en los territorios con iniciativas en 
materia de salud, educación, cultura, acceso y uso de TICs.

Un proceso similar vivió la cultura comunicativa del proyecto que fue transitando hacia la prodocomunicación, en la que toda la comunidad comparte el proceso abierto, en una apuesta mixta inclinada a crear red y conexiones entre el ecosistema de redes planetario, pero sin renegar a aparecer en los medios main stream. Los datos son elocuentes en cuanto a la eficacia de este modelo:

- Contenido propio. 190 textos publicados por FLOK en su site (floksociety.org), desde comunicaciones del proceso de investigación, hasta aportaciones de pensadores, colectivos, redes, activistas, alrededor del conocimiento libre.

- Contenido en medios. El número de impactos en medios tradicionales fue importante. En total, 103 medios del mundo, 56 ecuatorianos, 47 internacionales (incluímos blogs) han hablado del proceso FLOK. Otros 60, dieron espacio a la Cumbre del Buen Conocer (monitoreo realizado a principios de junio). Entre ellos, podrían destacarse TeleSur (Venezuela), El Diario (España), Página 12 (Argentina), Wired (Estados Unidos), Huffington Post Brasil, Le Monde Diplomatique (Chile), Hoy (República Dominicana) o El Comercio (Ecuador).

- Diálogo en redes sociales. Se usaron PADs abiertos y colaborativos, además de algunas herramientas propietarias, priorizando siempre que fuese posible las licencias libres. FLOK tiene perfiles en Twitter, quitter, Facebook, Flickr, Instagram, SlideShare, o Rebel Mouse, entre otras. Al final de la cumbre, la cuenta de Twitter de @FLOKsociety tenía en el medidor Klout (el principal para medir influencia) un valor de 63 (alto), igual que SENECYT e IAEN, instituciones como más historia y muchos más seguidores.

- Acciones. El equipo de comunicación de FLOK, considerando que la información en la era red es conexión, planeó diferentes acciones, campañas, actividades o encuentros orientadas al conocimiento libre y a reforzar el proceso. En la mayoría de ellas, se trabaja una mínima estrategia para visibilizar en redes el proceso, que en la mayoría de los casos acababa siendo Trending Topic en Twitter, trendsmap. Destacan, por su importancia conectiva, la Carta Abierta de los trabajadores del procomún, Minga Tecnológica o Minga en Red (WikiSprint), \#LiberaConocimiento.

- Redes-territorios. En la era red en la que la conexión a Internet en los territorios es cada vez más importante, es especialmente importante trabajar bajo el paradigma de territorios híbridos. Redes analógicas y digitales, convergiendo. Para ello, se pusieron en marcha los ya 
mencionados talleres de la Economía Social del Conocimiento (Infodesarrollo), los encuentros \#Beer2peer en los bares de Quito, la campaña sobre Eugenio Espejo Remix o la mencionada \#LiberaConocimiento.

Otro elemento considerable es la internacionalización del proyecto, que sobre todo ha partido del trabajo con movimientos sociales ecuatorianos y americanos: reuniones con el movimiento de okupación (Diabluma), wikisprint (Minga en Red) para mapear las experiencias colaborativas de Ecuador, en el que se crearon conexiones y del que sobrevive una lista de correos, sin ningún miembro de la dirección de FLOK. Se celebraron diferentes talleres y encuentros en El Diferencial (arte y tecnología en el Centro de Arte Contemporáneo). Se presentó FLOK en lugares tan diversos como la Contra Bienal de Arte de Cuenca, distintas universidades ecuatorianas, el MediaLab Prado (Madrid), Arena Net Mundial (espacio participativo del encuentro global Net Mundial de São Paulo, Brasil) o el Sharq Fórum (encuentro político social del mundo árabe y América Latina). En los últimos meses, este modelo se ha extendido con la participación en eventos en América Latina, como el Encuentro DxC (Pasto, Colombia), el 1er Congreso Internacional de Tecnopolítica y Derechos Humanos (Bogotá) y Europa, con el Encuentro de Economía Social en Atenas, el Open Everything en Dublín, la conferencia anual de software libre en Göteborg o la organización de la mesa de conocimiento en el Congreso de la Red de Economía Social en Zaragoza (España), a lo que hay que sumar la reciente participación dentro de las candidaturas municipalistas en España, la colaboración con Syriza (Grecia: http://floksociety.org/tag/syriza/) y Podemos (España) o exposiciones ante distintas comisiones del Parlamento Europeo.

\section{EFICACIA}

Dados los objetivos del proyecto, el impacto de la mirada FLOK y de las propuestas concretas sobre la sociedad civil y las instituciones ecuatorianas ha sido un objetivo fundamental, cuya valoración resulta ambivalente. A fin de reforzar la eficacia de los análisis de estas investigaciones hacia la economía social del conocimiento, adoptamos las siguientes acciones, más allá de los procesos de participación señalados,:

1. Delimitar el marco normativo y político que daba cobertura y aterrizaje nacional a las propuestas, sobre todo a partir de la Constitución de 2008 y del Plan Nacional de Buen Vivir 2013-2017.

2. Identificar las instituciones competentes para la implementación de las propuestas a través de distintos mecanismos (modificaciones normativas, diseño de política pública, 
proyectos productivos, etc.) e iniciar diálogos a partir de los primeros avances de investigación para establecer las estrategias de implementación.

3. Concretar los análisis de cada documento de política pública en un conjunto de recomendaciones para las instituciones competentes.

4. Trabajar en la exposición / traducción de estas propuestas para las instituciones y movimientos sociales interpelados. La fase central de este proceso ha consistido en la integración de los resultados de la investigación en el Código Orgánico de la Economía Social del Conocimiento (COESC) de la Secretaría Nacional de Ciencia (SENESCYT).

Por una parte, estamos contentos de la visibilidad que las nociones de ESC y de conocimiento común y abierto han adquirido en Ecuador, especialmente entre distintas instituciones dedicadas al desarrollo y a la mejora del talento humano, movimientos tecnopolíticos y algunos espacios académicos. A su vez, muchos contenidos forman parte del borrador de la ley orgánica de esa ESC que se está tramitando en la Asamblea Nacional y de las políticas de investigación o de divulgación científica. Incluso uno de los prototipos diseñado dentro de FLOK, como el de educación masiva online (MOOC) en la universidad postgrado del estado (UPEx) ha iniciado su actividad con distintos cursos y una capacidad de formación y ahorro inédita en la formación continua del país. Por otra parte, la eficacia de las recomendaciones políticas y de los proyectos productivos en muchas áreas requiere una concreción y una atención a la implementación que un equipo disminuido al $10 \%$ desde julio de 2014 no ha podido desarrollar. Por ejemplo, los responsables políticos advierten las ventajas que nuestras recomendaciones detallan de una migración de los escritorios a software libre pero el proceso requiere de planes de formación, de programas piloto y de tácticas concretas que solventen los cuellos de botella que nuestras recomendaciones han encontrado, de modo que todo ello requeriría profundizar los procesos que hemos iniciado en estos nueve meses.

El futuro del proyecto está marcado por esta doble necesidad. Concretar y hacer viables procesos de reforma de la administración pública y proyectos productivos respecto a los que el éxito parece asegurado pero que no se pueden ejecutar sin profundizar la apuesta por el conocimiento libre. A la vez que es imprescindible reforzar los discursos y las hojas de ruta de la mayor ambición teórica e internacionalista que aseguren que la transición de Ecuador y de la Región hacia la economía del conocimiento se produce bajo esas condiciones de acceso, utilidad social y sostenibilidad que harán conquistar a estos países su verdadera 
libertad y justicia social en una ecnomía globalizada dominada por la distribución desigual del conocimiento y la jerarquía tecnológica. Movimientos sociales en todo el mundo y algunas pocas instituciones públicas hacen indefectible esta tendencia de la que FLOK Society es solo un catalizador en América Latina.

Una versión ampliada y detallada de esta valoración puede encontrarse en http://floksociety.org/2014/11/10/2737/ 